\title{
Odonatos de la Sierra Nevada de Santa Marta, Colombia: una lista preliminar
}

\author{
(ㄱ) Melizza Tobias-Loaiza*, () Cesar E. Tamaris-Turizo
}

Grupo de Investigación en Biodiversidad y Ecología Aplicada, Universidad del Magdalena, Santa Marta, Colombia

\begin{abstract}
Resumen
Se presenta una lista de especies y géneros de odonatos de ocho ríos de la Sierra Nevada de Santa Marta (SNSM) producto de la revisión de artículos científicos, trabajos de grado y especímenes depositados en el centro de Colecciones Biológicas de la Universidad del Magdalena (CEBUMAG). Se registran 38 especies distribuidos en 35 géneros en ocho familias. La familia Libellulidae presentó la mayor riqueza (14 géneros) y los rangos más amplios de distribución altitudinal (entre 50 y $2800 \mathrm{msnm}$ ), encontrándose en todas las coberturas vegetales. El río Gaira exhibió la mayor riqueza entre los ríos revisados, 26 géneros, de los cuales ocho son exclusivos de la cuenca, en tanto que en el río Ranchería solo se registró Progomphus. La distribución espacial más amplia fue la de los géneros Argia y Brechmorhoga, registrados en seis ríos. La revisión evidenció la falta de estudios en las vertientes oriental y suroccidental de la Sierra Nevada de Santa Marta, por lo que se recomienda realizar trabajos taxonómicos y de biodiversidad para contribuir al conocimiento de odonatos en el Caribe colombiano. (c) 2019. Acad. Colomb. Cienc. Ex. Fis. Nat.
\end{abstract}

Palabra clave: Libélula; Caballito del diablo; Distribución; Magdalena.

Odonata of the Sierra Nevada de Santa Marta, Colombia: A preliminary list

\begin{abstract}
We raised a list of Odonata species and genera from eight rivers of the Sierra Nevada of Santa Marta (SNSM) after the revision of scientific articles, theses, and specimens deposited in the Centro de Colecciones de la Universidad del Magdalena (CEBUMAG). We found 38 species distributed in 35 genera and eight families. The Libellulidae family presented the greatest richness (14 genera) and the widest range of altitudinal distribution (between 50 and $2,800 \mathrm{~m}$ a.s.1.) found in all vegetable coverages. The Gaira River exhibited the greatest wealth among the revised rivers with 26 genera of which eight are exclusive to the basin while in the Ranchería River only Progomphus was registered. Argia and Brechmorhoga had a wide spatial distribution as they were recorded in six rivers. The review revealed the lack of studies on the eastern and southwestern slopes of the Sierra Nevada de Santa Marta, so it is recommended to carry out taxonomic and biodiversity work to contribute to the knowledge of Odonata in the Colombian Caribbean. (C) 2019. Acad. Colomb. Cienc. Ex. Fis. Nat.
\end{abstract}

Key words: Dragonfly; Damselfly; Distribution, Magdalena.

\section{Introducción}

Los odonatos son insectos hemimetábolos, pues presentan los estados de huevo, larva y adulto (Arango \& Roldán, 1983; Roldán, 1988). Los huevos y las larvas se desarrollan en diferentes ambientes como ríos, lagos, pozos, pantanos, manglares e, incluso, en el agua acumulada en las plantas (fitotelmata), mientras que los adultos habitan en la vegetación cerca a los cuerpos de agua (Roldán, 1988; GómezAnaya, 2008). El ciclo de vida de la larva puede durar desde pocos meses hasta más de un año, según la especie, el clima, el hábitat y la disponibilidad de alimento (Arango \& Roldán, 1983; Ramírez, 2010).

En los ecosistemas de agua dulce los odonatos son importantes porque actúan como control biológico de insectos vectores de enfermedades tropicales (López, et al., 1998).
Además, tienen un papel fundamental en la transferencia de energía (desde los niveles intermedios hasta el superior), por lo que son considerados como un eslabón intermedio o superior (en ausencia de organismos como peces y anfibios) en las redes tróficas (Santos, et al., 1981). Este grupo de insectos es utilizado como indicador de alteraciones ambientales debido a las exigencias ambientales que tienen algunas especies durante sus estadios inmaduros, los cuales se desarrollan en el medio acuático, y la dependencia de los adultos de una estructura vegetal compleja que les permita completar su ciclo de vida (Gómez-Anaya, 2008; Sánchez,

*Correspondencia:

Melizza Tobias-Loaiza; melizzatobias.26@gmail.com

Recibido: 31 de enero de 2019

Aceptado: 5 de abril de 2019

Editor: Elizabeth Castañeda 
et al., 2009). Por lo tanto, los factores de estrés que afectan los sistemas acuáticos y ribereños tienen efectos directos e indirectos en la dinámica de las poblaciones de odonatos.

En Colombia se han realizado trabajos que compilan información de los inventarios locales y revisiones de material depositado en centros de colecciones biológicas, entre los que sobresalen las investigaciones de Palacino-Rodríguez (2009), quien realizó un inventario de Anisoptera a partir de especímenes recolectadas desde 1940 en 27 departamentos del país cuyo principal aporte fue el registro de 91 especies. Posteriormente, Pérez-Gutiérrez \& Palacino-Rodríguez (2011) presentaron lo que en su momento sería considerado la lista más completa de odonatos, con 335 especies y 90 géneros. Más adelante, Bota-Sierra, et al. (2016) evaluaron el estado de conservación de las libélulas en los Andes tropicales e indicaron que el número de especies registrado para Colombia había aumentado a 389. Actualmente, en el país se han registrado aproximadamente 434 especies, principalmente por las contribuciones de Bota-Sierra, et al. (2018a, 2018b).

En el río Gaira (Sierra Nevada de Santa Marta) se registran 26 especies de odonatos en trabajos como el de PérezGutiérrez (2003), el cual incluyó un estudio biotaxonómico en el distrito de Santa Marta y en ríos que descienden de la Sierra Nevada de Santa Marta, y el de Tobías-Loaiza (2016), en el que se evaluó la diversidad de odonatos en la parte media del río Gaira. Además, se han hecho trabajos aislados que involucran a las comunidades de macroinvertebrados acuáticos a nivel de géneros en otras zonas de la Sierra Nevada de Santa Marta (Tamaris-Turizo, et al., 2013; Rúa-García, et al., 2015; Barragán, et al., 2017). Por ello, es necesario desarrollar estudios que recopilen la información dispersa sobre este grupo biológico, con el fin de comprender mejor su dinámica espacial y contribuir al estudio de este orden de insectos en el Caribe colombiano.

\section{Materiales y métodos}

Area de estudio. La Sierra Nevada de Santa Marta (Sierra Nevada de Santa Marta) es un macizo montañoso ubicado al norte de Colombia, entre los $10^{\circ} 01^{\prime} 05^{\prime \prime}, 11^{\circ} 20^{\prime} 11^{\prime \prime} \mathrm{N}$

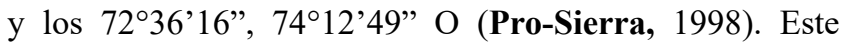
sistema montañoso es de gran importancia para la región Caribe dado que se trata de una fuente hídrica por la que corren 29 ríos principales (Pro-Sierra, 1998). En esta formación, la vertiente norte bordea el mar Caribe desde el sur de La Guajira hasta Santa Marta, la vertiente occidental limita con la Ciénaga Grande de Santa Marta, la planicie del río Magdalena y el mar Caribe y la vertiente oriental está rodeada por el río Cesar y el río Ranchería. Cada flanco de la Sierra Nevada de Santa Marta presenta un clima diferente, determinado por factores como la exposición al mar y los vientos del nordeste (Pro-Sierra, 1998; Instituto Geográfico Agustín Codazzi-IGAC, 1993; Unidad Administrativa Especial Parques Nacionales Naturales de Colombia-UAESPNN, 2005).
En los ríos estudiados se encuentran siete formaciones vegetales, las cuales se localizan desde el nivel del mar hasta la parte más elevada de las cuencas. Según la clasificación sugerida por Holdridge y adaptada por Espinal \& Montenegro (1963) para Colombia, en los ríos de la Sierra Nevada de Santa Marta se registran las formaciones vegetales: monte espinoso tropical ( 0 -100 m s.n.m.), bosque muy seco tropical (100-200 m s.n.m.), bosque seco tropical (200-700 m s.n.m.), bosque húmedo subtropical (700-900 $\mathrm{m}$ s.n.m.), bosque muy húmedo subtropical (900-1.700 m s.n.m.), bosque muy húmedo montano bajo (1.700-2.350 m s.n.m.) y bosque premontano (2.350-2.850 m s.n.m.).

Análisis de los datos. La lista de odonatos se recopiló a partir de la información obtenida de artículos científicos, trabajos de pregrado y mediante la consulta de los ejemplares depositados en el Centro de Colecciones Biológicas de la Universidad del Magdalena (CEBUMAG). Las muestras provienen principalmente de estudios de diversidad y de ecología de macroinvertebrados bentónicos en diferentes ríos de la Sierra Nevada de Santa Marta.

A partir de la revisión de la literatura, se elaboró una matriz con información del número de organismos por familias, géneros y especies, en la cual se registró la siguiente información: nombre de la localidad, coordenadas, altitud y formación vegetal según la clasificación de Espinal \& Montenegro (1963). Con base en dicha matriz, se elaboraron gráficas de distribución altitudinal para asociar la presencia de los géneros con la altitud y con la formación vegetal correspondiente.

\section{Resultados}

Como producto de la revisión, se presenta una lista de 38 especies distribuidas en 35 géneros y ocho familias de odonatos de ocho ríos de la Sierra Nevada de Santa Marta: Gaira, Manzanares, Toribio, Córdoba, Palomino, Piedra, Ranchería y Guatapurí (Tabla S1, https://www.raccefyn.co/ index.php/raccefyn/article/downloadSuppFile/832/3841). La familia Libellulidae fue la más representativa, con 14 géneros, seguida de la Coenagrionidae, con siete, en tanto que la Platystictidae y la Calopterygidae presentaron un género cada una (Tabla S1, https://www.raccefyn.co/index. php/raccefyn/article/downloadSuppFile/832/3841).

En cuanto a la distribución altitudinal, Libellulidae presentó los rangos más amplios de distribución (entre 50 y $2.800 \mathrm{~m}$ s.n.m.), mientras que Lestidae, la menor (entre los 600 y los 900 m s.n.m.) (Figura 1). Los géneros con mayor distribución fueron Erythrodiplax, Hetaerina y Sympetrum, y entre los de menor distribución se encontraron Acanthagrión, Agriogomphus y Telebasis. Se destaca que Archaeogomphus, Coryphaeshna, Enallagma, Gynacantha, Miathyria, Micrathyria, Perithemis y Triacanthagyna solo se registraron en un solo sitio. La mayor riqueza de géneros se registró en los tramos medios y bajos de los ríos (Figura 2).

Con respecto a la distribución espacial, el río Gaira exhibió la mayor riqueza, con 26 géneros, de los cuales ocho son exclusivos, seguido del río Manzanares, con 17 géneros, 


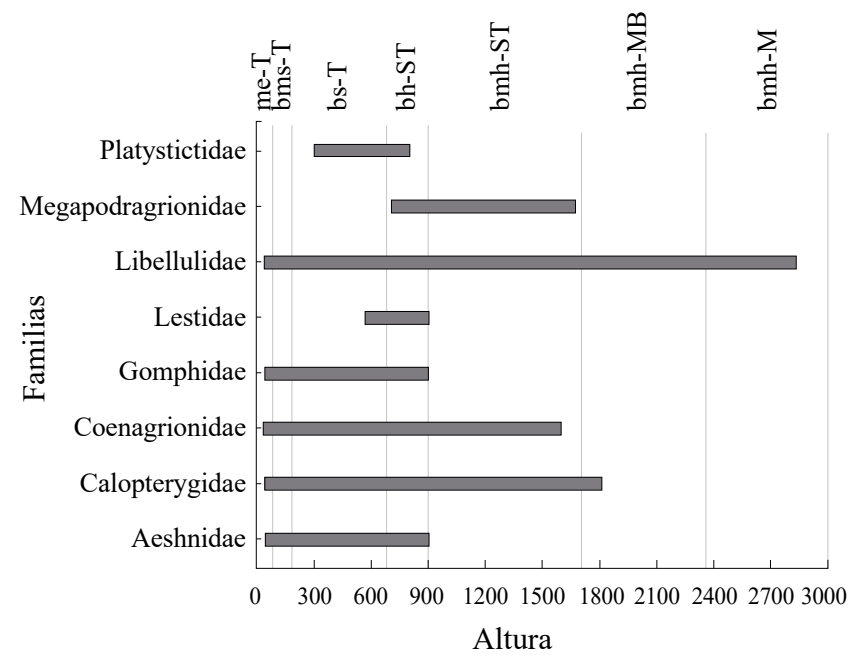

Figura 1. Distribución altitudinal de las familias del orden Odonata en sistemas fluviales de la Sierra Nevada de Santa Marta de los cuales tres son exclusivos. Por el contrario, los ríos Ranchería y Palomino registraron las menores riquezas, con uno y cuatro géneros, respectivamente. Los géneros Argia y Brechmorhoga tuvieron la mayor distribución espacial, pues se encontraron en seis de los ochos ríos analizados, mientras que los ejemplares de Coryphaeshna, Gynacantha, Rhionaeschna, Triacanthagyna, Acanthagrion, Enallagma, Mecistogaster, Psaironeura, Archaeogomphus, Archilestes, Erythrodiplax, Miathyria, Orthemis, Perithemis y Teinopodagrion se capturaron en un solo lugar (Figura 3).

Con relación a las coberturas vegetales, Libellulidae estuvo en todos los tipos de bosques estudiados, desde el bosque espinoso tropical (be-T) hasta el bosque muy húmedo montano (bmh-M), a diferencia de lo observado en Platystictidae y Lestidae, que solo estuvieron presentes en el bosque seco tropical (bs-T) y el bosque húmedo subtropical (bh-ST) (figura 1). Hetaerina y Sympetrum se registraron en seis tipos de coberturas, desde el be-T hasta el bosque

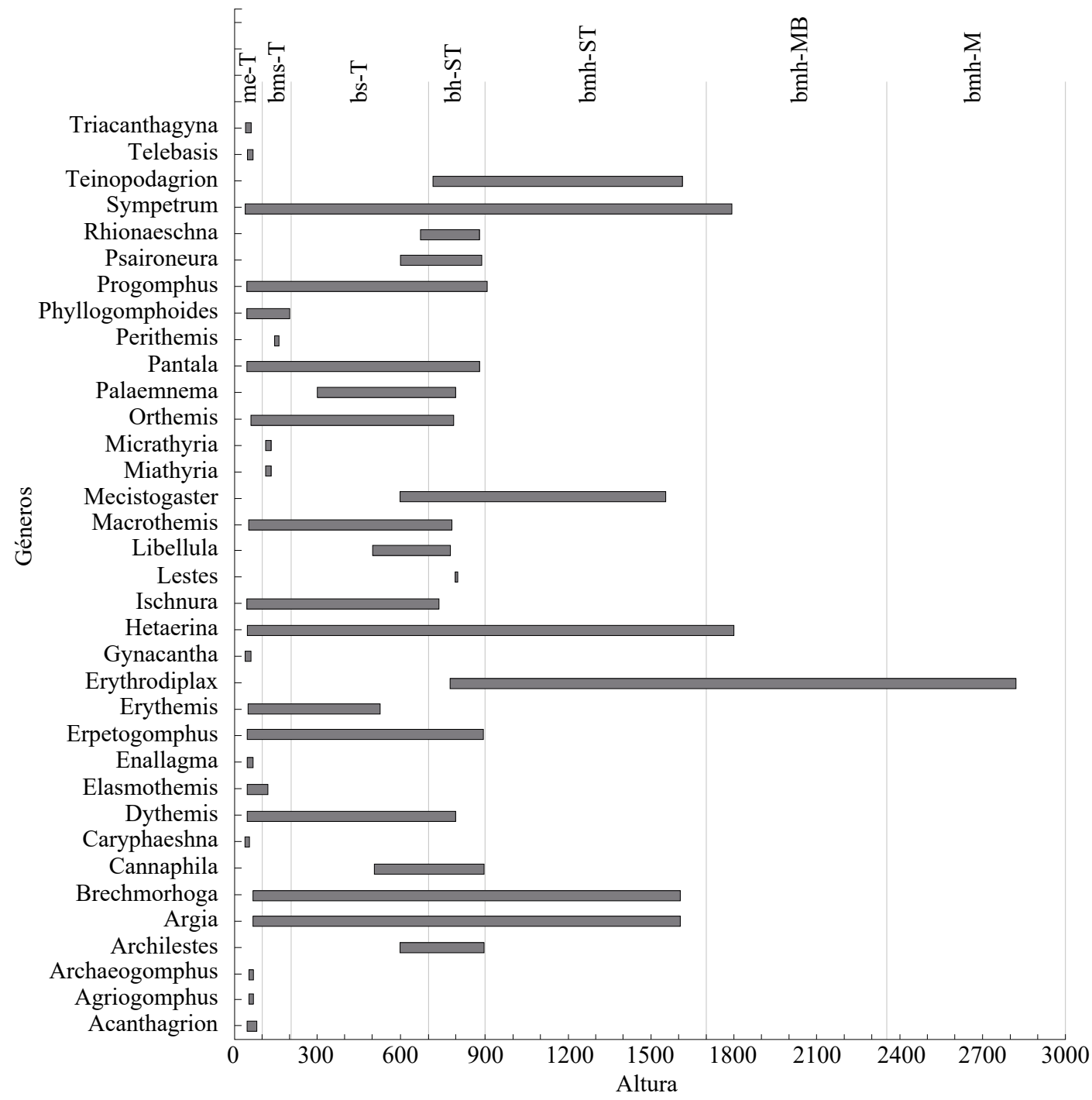

Figura 2. Distribución altitudinal de los géneros del orden Odonata en sistemas fluviales de la Sierra Nevada de Santa Marta 


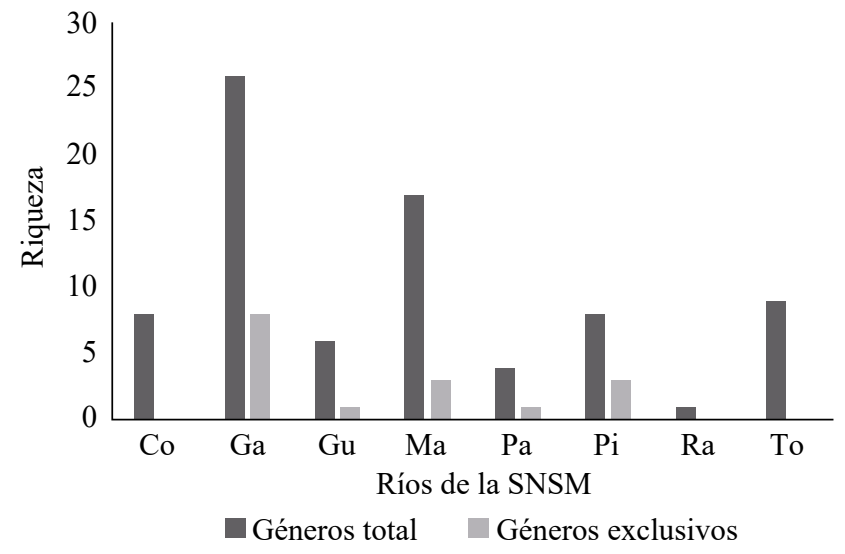

Figura 3. Riqueza de géneros en ocho ríos de la Sierra Nevada de Santa Marta. Co: río Córdoba, Ga: río Gaira, Gu: río Guatapurí, Ma: río Manzanares, Pa: río Palomino, Pi: río Piedra, Ra: río Ranchería y To: río Toribio

muy húmedo montano bajo (bmh-MB). En contraste, Rhionaeschna solo se encontró en el bh-ST. En todos los casos de géneros con el menor rango de distribución, los ejemplares fueron capturados en localidades exclusivas ubicadas principalmente en el be-T y el bms-T (Figura 2).

\section{Discusión}

La revisión realizada refleja que hay poca riqueza de especies y de géneros de Odonata en la Sierra Nevada de Santa Marta en comparación con la registrada en trabajos realizados en otras regiones de Colombia, como el de Bota-Sierra, et al. (2015), en el cual se hizo una amplia revisión en la Amazonia colombiana y se registraron 108 especies distribuidas en 55 géneros y 15 familias, y el de Bota-Sierra, et al. (2018a), en el cual se llevó a cabo un trabajo de campo intensivo en los departamentos de Cauca, Nariño y Putumayo, y se registró una riqueza de 68 especies distribuidas en 39 géneros y 12 familias. Es muy probable que el número de especies y géneros en la Sierra Nevada de Santa Marta aumente en la medida de que se realicen más trabajos taxonómicos a nivel de especie en zonas donde se han hecho pocos estudios, como en la vertiente de la Sierra que colinda con La Serranía del Perijá (sur de La Guajira).

La gran riqueza de géneros exhibida por Libellulidae se corresponde con su gran diversidad y su capacidad para adaptarse en áreas con alto grado de intervención antrópica (Carle, 1979; Bermúdez, 2005). Los resultados de esta revisión coinciden con lo registrado en otros trabajos realizados en Colombia (Arango \& Roldán, 1983; Altamiranda, 2009; Garzón-Sanabria \& Realpe, 2009 y Altamiranda, et al., 2010) y en el Neotrópico (Novelo-Gutiérrez \& Gómez-Anaya, 2009). Por el contrario, la poca riqueza de géneros de Calopterygidae y Platystictidae concuerda con sus distribuciones biogeográficas, pues el único género de la familia Calopterygidae al oeste de los Andes es Hetaerina y la familia Platystictidae solo está representada por el género Palaemnema en el Neotrópico (Garrison, et al., 2010).
La disminución en la riqueza de géneros de odonatos en la medida que incrementa la altitud, se ha documentado en América del Norte (Corbet, 1999), Sudafrica (Samways, 1989), Europa central (Maibach \& Meier, 1987), en el Himalaya (Mahato \& Edds, 1993) y en Colombia (Arango \& Roldán 1983; Bota-Sierra, 2014). Según McCoy (1990), la diversidad de insectos es limitada en las alturas, debido a las rigurosas condiciones del clima y a la disminución de los recursos, en tanto que la riqueza es mayor en las partes medias y bajas de las cuencas, lo que también puede estar relacionado con la heterogeneidad de coberturas vegetales en estas zonas.

En Colombia, la familia con mayor rango altitudinal es Aeshnidae, seguida de Libellullidae y Coenagrionidae (Bota-Sierra, 2014). Sin embargo, en este trabajo la familia Libellulidae presentó mayor rango altitudinal, como se ha registrado en otros estudios realizados en Colombia (Arango \& Roldán, 1983) y en otros países como España (Ocharán \& Torralba, 2004; Mezquita-Aranburu, 2016). Libellulidae se caracteriza por presentar una amplia distribución altitudinal, debido a los hábitos migratorios que tienen algunos géneros como Pantala y Miathyria (Srygley, 2003; Kalkman, 2008; Bermúdez \& López-Victoria, 2009, Paulson, 2009) y por la capacidad que tienen otros géneros de adaptarse a ecosistemas de alta montaña como Sympetrum y Erythrodiplax (Bota-Sierra, 2014); además, está presente en todos los países neotropicales (Kalkman, et al., 2008). Otros factores que pueden influir en su distribución son la habilidad de los adultos de desarrollar un vuelo rápido, que les permite desplazarse a largas distancias, y la capacidad para colonizar diversos cuerpos de agua y coberturas vegetales de algunas especies euritolerantes (Arango \& Roldán, 1983; Srygley, 2003; Esquivel, 2006). Ello explica que esta familia se haya encontrado en todos los tipos de coberturas comprendidos desde el nivel del mar hasta los 2.800 m s.n.m.

Por el contrario, Platystictidae y Lestidae presentaron los menores rangos de distribución, debido a que los adultos de Platystictidae tienen hábitos muy restringidos, se encuentran normalmente en el sotobosque y tienen un periodo de vuelo corto (Ramírez, 2010; González-Soriano \& NoveloGutiérrez, 2014). Por su parte, Lestidae se encuentra en áreas abiertas y sus larvas se pueden encontrar en aguas lentas, remansos de arroyos, pantanos o pozas temporales (Ramírez, 2010), sin embargo, es una familia con tolerancia moderada a las condiciones climáticas, por lo que es más común en altitudes bajas (Samways, 1989). En países como Costa Rica, las familias Platystictidae y Lestidae se han encontrado desde el nivel del mar hasta los $1.500 \mathrm{~m}$ (Ramírez, 2010).

El amplio gradiente altitudinal presentado por Erythrodiplax, Hetaerina y Sympetrum, se debe a que son géneros que se pueden encontrar en diversos hábitats. Sympetrum y Erythrodiplax se han registrado en varios tipos de cuerpos de agua lénticos, como pantanos, lagos, 
estanques, charcas temporales y arroyos con poca corriente, mientras que Hetaerina lo hace principalmente en corrientes rápidas (Garrinson, et al., 2006). Estos géneros se encuentran comúnmente en zonas con alto grado de intervención antrópica y se adaptan a diferentes tipos de coberturas (Altamiranda, 2009).

Acanthagrión y Telebasis presentaron los menores rangos de distribución altitudinal. Sin embargo, estos géneros se encuentran distribuidos ampliamente en el Neotrópico (Alonso-Eguía, et al., 2002), donde son muy comunes en zonas bajas y se pueden encontrar en aguas estancadas, tallos de plantas flotantes y pantanos. En el caso de Telebasis, es común encontrar ejemplares en marismas, pero, aparentemente, están ausentes en los ríos (Garrinson, et al., 2006). En esta revisión, Telebasis se registró en zonas de desembocadura.

En cuanto a la distribución espacial, todos los géneros de esta lista estuvieron presentes en la vertiente noroccidental, en especial en los ríos Gaira y Manzanares, donde se han realizado la mayoría de los estudios con macroinvertebrados acuáticos. Pocos han sido los trabajos realizados en otras vertientes de la Sierra Nevada de Santa Marta, por lo que en esta revisión se registró poca riqueza de géneros en la vertiente suroccidental y su ausencia en la oriental.

\section{Conclusiones}

En este trabajo se evidencia que la Sierra Nevada de Santa Marta tiene una gran riqueza de géneros de libélulas, en especial en la vertiente noroccidental. Sin embargo, es necesario realizar más estudios en las vertientes suroccidental y oriental enfocados en la taxonomía y en la asociación de larvas y adultos para llevar a cabo su identificación hasta el nivel de especie. Es posible que con más estudios en estas zonas, aumente el número de géneros presentes en la Sierra Nevada de Santa Marta y se obtenga una lista más completa de odonatos para la región.

\section{Información suplementaria}

Tabla S1. Lista de especies registradas en ríos de la Sierra Nevada de Santa Marta. Co: río Córdoba, Ga: río Gaira, Gu: río Guatapurí, $\mathrm{Ma}$ : río Manzanares, Pa: río Palomino, Pi: río Piedra, $\mathrm{Ra}$ : río Ranchería y To: río Toribio. *Especies capturadas en estado adulto. Vea la tabla S1 en: https://www.raccefyn.co/index.php/raccefyn/ article/downloadSuppFile/832/3841

\section{Agradecimientos}

Agradecemos a la Colección Biológica de la Universidad del Magdalena, por facilitar la información complementaria para la realización de este documento. A Alonso Ramírez y Rodolfo Novelo-Gutiérrez, por la confirmación de la identificación de las especies, y a los evaluadores por sus grandes aportes y comentarios acertados, que sin duda mejoraron la calidad del manuscrito.

\section{Contribución de los autores}

Los dos autores participaron en el proceso de conceptualización de la revisión, tratamiento de la información y los datos y escritura del manuscrito.

\section{Conflicto de intereses}

Los autores declaran no tener conflictos de intereses.

\section{Referencias}

Aguirre-Pabón, J., Barrios, J. R., y Ospina-Torres, R. (2012). Deriva de macroinvertebrados acuáticos en dos sitios con diferente grado de perturbación, río Gaira, Santa MartaColombia. Intropica: Revista del Instituto de Investigaciones Tropicales. 7 (1): 1.

Alonso-Eguía, L., González-Soriano, E., Gutiérrez-Yurrita, P. J. (2002). Listado y distribución de los odonatos de la cuenca del Río Moctezuma, Centro-Occidente de México (Insecta: Odonata). Folia Entomológica Mexicana. 41 (3): 347-357.

Altamiranda, M. (2009). Diversidad de libélulas (insecta-odonata) para dos usos de suelo, en un bosque seco tropical. Revista Facultad Nacional de Agronomía Medellín. 62 (2): 50715079.

Altamiranda, M., Pérez, L., Gutiérrez, L. (2010). Composición y preferencia de microhábitat de larvas de Odonata (Insecta) en la Ciénaga San Juan de Tocagua (Atlántico, Colombia). Caldasia. 32 (2): 1-13.

Arango, M. C. \& Roldán, G. (1983). Odonatos inmaduros del departamento de Antioquia en diferentes pisos altitudinales. Actualidades Biológicas. 12 (46): 91-105.

Barragán, M. F., Tamaris-Turizo, C. E., Rúa-García, G. A. (2017). Comunidades de insectos acuáticos de los tres flancos de la Sierra Nevada de Santa Marta, Colombia. Biota Colombiana. 17 (2): 47-61.

Bermúdez Rivas, C. (2005). Clave para los imagos de los géneros de Libellulidae (Odonata: Anisoptera) del Valle del Cauca, Colombia. Boletín del Museo de Entomología de la Universidad del Valle. 6 (1): 7-22.

Bermúdez, C. R. \& López-Victoria, M. (2009). Primeros registros de libélulas (Odonata: Anisoptera) en la Isla Malpelo, Colombia/First records of dragonflies (Odonata: Anisoptera) of Malpelo Island, Colombia. Revista Colombiana de Entomología. 35 (2): 286.

Bota-Sierra, C.A., C. Moreno-Arias, Faasen, T. (2015). Preliminary list of Odonata from the Colombian Amazon, with descriptions of Inpabasis nigridorsum sp. nov. y Diaphlebia richteri sp. nov. (Coenagrionidae y Gomphidae). International Journal of Odonatology. 18 (3): 249-268.

Bota-Sierra C. A. (2014). A brief look at the Odonata from the Páramo ecosystems in Colombia, with the descriptions of Oxyallagma colombianum sp. nov. and Rhionaeschna caligo sp. nov. (Odonata: Coenagrionidae, Aeshnidae, Libellulidae). Zootaxa. 3856 (2): 192-210.

Bota-Sierra, C. A., Maufray, B., Palacino-Rodríguez, F., Hofmann, J., Tennessen, K., Rache, L., Tognelli, M. F. (2016). Estado de conservación de las libélulas de los Andes Tropicales. En Tognelli, M. F., Lasso, C. A., Bota-Sierra, C. A., Jiménez-Segura, L. F., y Cox, N. A. (Editores). Estado de conservación y distribución de la biodiversidad de agua dulce en los Andes Tropicales. UICN. Gland, Cambridge y Arlington: UICN. 
Bota-Sierra, C. A., Corso, A., Janni, O., Sandoval-H, J., Viganò, M. (2018a). First dragonfly records from Colombia and the confirmation of the synonymy of Philogenia monotis and $P$. tinalandia (Insecta: Odonata). International Journal of Odonatology. 21 (2):115-127.

Bota-Sierra, C. A., Sánchez-Herrera, M., Palacino-Rodríguez, F. (2018b). Odonata from protected areas in Colombia with new records and description of Cora verapax sp. nov. (Zygoptera: Polythoridae). Zootaxa. 4462 (1):115-131.

Carle, F.L. (1979). Environmental monitoring potential of the Odonata, with a list of rare and endangered Anisoptera of Virginia, United States. Odonatologica. 8 (4): 319-323.

Corbet, P. S. (1999). Dragonflies, Behaviour and Ecology of Odonata. Colchester, England: Harley Books. p. 829.

Espinal, T. \& Montenegro, L.S. (1963). Formaciones vegetales de Colombia. Memoria explicativa del mapa ecológico. Bogotá, Colombia, IGAC-Instituto Geográfico Agustín Codazzi. p. 201.

Esquivel, C. (2006). Libélulas de Mesoamerica y el Caribe. San José de Costa Rica: Editorial INBIO. p. 319.

Eyes-Escalante, M., Rodríguez-Barrios, J., Gutiérrez-Moreno, l. C. (2012). Descomposición de la hojarasca y su relación con los macroinvertebrados acuáticos del río Gaira (Santa Marta-Colombia). Acta Biológica Colombiana. 17 (1): 77-92.

Garrison, R.W., Von Ellenrieder, N., Louton, J. A. (2006). Dragonfly Genera of the New World: An illustrated and annotated key to the Anysoptera. Baltimore: Johns Hopkins University Press. p. 368.

Garrison, R.W., Von Ellenrieder, N., Louton, J. A. (2010). Damselfly Genera of the New World: An illustrated and annottated key to the Zygoptera. Baltimore: Johns Hopkins University Press. p. 490.

Garzón-Sanabria, C. \& Realpe, E. (2009). Diversidad de Odonata (Insecta) en la reserva natural Cabildo-Verde (Sabana de Torres-Santander, Colombia), una aproximación hacia la conservación. Caldasia. 31 (2): 459-470.

González-Soriano, E. \& Novelo-Gutiérrez, R. (2014). Biodiversidad de Odonata en México. Revista mexicana de biodiversidad. 85: 243-251.

Gómez-Anaya, J. A. (2008). Ecología de Los ensambles de larvas de odonatos (Insecta), y su uso potencial como indicadores de calidad ecológica en la Sierra de Coalcoman, Michoacán, México. Tesis para obtener el título de doctor en Recursos Bióticos. Instituto de Ciencias Básicas e Ingeniería. Universidad Autónoma de Estado de Hidalgo.

Granados-Martínez, C. E. (2013). Análisis de la dieta de los macroinvertebrados bentónicos en un gradiente altitudinal de la cuenca del río Gaira (Sierra Nevada de Santa MartaColombia). Universidad del Zulia. Facultad Experimental de Ciencias. Trabajo de Grado Magister Scientiarum en Ciencias Biológicas Mención Ecología Acuática.

Instituto Geográfico Agustín Codazzi - IGAC. (1993). Proyecto Piloto de Ordenamiento Territorial de la Sierra Nevada de Santa Marta, Una Aproximación Metodológica. Santa Fe de Bogotá: IGAC.

Kalkman, V. J., Clausnitzer, V., Dijkstra, K. D., Orr, A. G., Paulson, D. R., van Tol, J. (2008). Global diversity of dragonflies (Odonata) in freshwater. Hidrobiologia. 595: 351-363.
López, D., Espinoza, P., López, M., Valle, S., Rivera, P., García, I. (1998). Las libélulas (Insecta-Odonata) como biorreguladores de larvas de mosquitos en Nicaragua. Revista Nicaraguense de Entomologia. 45: 1-5.

Mahato, M. \& Edds, D. (1993). Altitudinal distribution of odonate larvae in Nepal's Gandaki river. Odonatologica. 22: 213-221.

Maibach, A., Meier, C., Dufour, C. (1987). Atlas de distribution des libellules de Suisse (Odonata) (avec liste rouge). Centre suisse de cartographie de la faune, Neuchâtel.

McCoy, E. D. (1990). The distribution of insects along elevational gradients. Oikos. 58: 313-332.

Manjarrez-García, G. \& Manjarrez-Pinzón, G. (2004). Contribución al conocimiento hidrobiológico de la parte baja de los ríos de la vertiente noroccidental de la Sierra Nevada de Santa Marta, Colombia. Intropica. 1: 39.

Martínez, G. (2010). Macroinvertebrados acuáticos como sistema de evaluación de contaminación del balneario Hurtado, río Guatapurí, Valledupar-Cesar. Trabajo de grado de Especialista en Química Ambiental. Universidad Industrial de Santander.

Mezquita-Aranburu, I. (2016). Primera cita de Sympetrum sanguineum (Müller, 1764) (Odonata, Libellulidae) para Gipuzkoa (País Vasco, España). Munibe Ciencias Naturales. 64: 161-165.

Novelo-Gutiérrez, R. y Gómez-Anaya J.A. (2009). A comparative study of Odonata (Insecta) assemblages along an altitudinal gradient in the sierra de Coalcomán Mountains, Michoacán, México. Biodiversity and Conservation. 18: 679-698.

Ocharán, F. J. \& Torralba-Burrial, A. (2004). La relación entre los odonatos y la altitud: el caso de Asturias (Norte de España) y la Península Ibérica (Odonata). Boletín de la Sociedad Entomológica Aragonesa. 35: 103-116

Palacino-Rodríguez, F. (2009). Dragonflies (Odonata: Anisóptera) of the collection of the Instituto de Ciencias Naturales, Universidad Nacional de Colombia. Boletín del Museo de Entomología de la Universidad del Valle. 10 (1): 37-41.

Paulson, D. R. (2009). Dragonflies and damseflies of the West. USA: Princenton University Press. p. 535.

Pérez-Gutiérrez, L. A. (2003). Estudio biotaxonómico de los odonatos (Insecta: Odonata Fabricius.1793) del distrito de Santa Marta (Magdalena-Colombia). Tesis de pregrado para optar el título de Biólogo. Universidad del Magdalena. Facultad de Ciencia Básica. Colombia.

Pérez-Gutiérrez, L.A. \& Palacino-Rodríguez, F. (2011). Updated checklist of the Odonata known from Colombia. Odonatologica. 40 (3): 203.

PROSIERRA - Fundación Pro-Sierra Nevada De Santa Marta. (1998). Evaluación ecológica rápida: definición para áreas críticas para la conservación en la Sierra Nevada de Santa Marta. Santa Marta: Ministerio del Medio Ambiente, UAESPPNN The Nature Conservacy -USAID- Embajada de Japón.

Ramírez, A. (2010). Capítulo 5: Odonata. Revista de Biología Tropical. 58: 97-136.

Roldán, G. (1988). Guía para el estudio de los macroinvertebrados acuáticos del departamento de Antioquia. Universidad de Antioquia, Fondo FEN. Medellín, Colombia.

Rodríguez-Barrios, J. A. (2011). Descriptores funcionales en un sistema fluvial de montaña. Santa Marta, Colombia. Disertación doctoral. Universidad Nacional de Colombia. 
Rúa-García, G., Tamaris-Turizo, C., Zúñiga, M. D. (2015). Composición y distribución de los órdenes Ephemeroptera, Plecoptera y Trichoptera (Insecta) en ríos de la Sierra Nevada de Santa Marta, Colombia. Revista de Ciencias. 19 (2): 11-29.

Samways, M. J. (1989). Taxon turnover in Odonata across a 3000 $\mathrm{m}$ altitudinal gradient in southern Africa. Odonatologica. 18: $263-274$.

Sánchez, A., Pérez, J., Jiménez, E., Tovar, C. (2009). Los Odonatos de Extremadura. Consejería de industria, energía y medio ambiente. Junta de Extramadura, Mérida. p. 344.

Santos, N.D. (1981). Odonata. En: Hurlbert, s.h. et al. Aquatic Biota of Tropical South America. Part I. Arthropoda. San Diego State University, San Diego, California. p. 323.

Srygley, R. B. (2003). Wind drift compensation in migrating dragonflies Pantala (Odonata: Libellulidae). Journal of Insect Behavior. 16 (2): 217-232.
Tamaris-Turizo, C., Rodríguez-Barrios, J., Ospina-Torres, R. (2013). Deriva de macroinvertebrados acuáticos a lo largo del río Gaira, vertiente noroccidental de la Sierra Nevada de Santa Marta, Colombia. Caldasia. 35 (1): 149-163.

Tobías-Loaiza, M. (2016). Comunidades del orden Odonata en la parte media del río Gaira, Sierra Nevada De Santa Marta, Colombia. Tesis de pregrado para optar el título de Biólogo. Universidad del Magdalena. Facultad de Ciencia Básica. Colombia.

Unidad Administrativa Especial Parques Nacionales Naturales de Colombia-UAESPNN. (2005). Plan de Manejo Parque Nacional Natural Sierra Nevada de Santa Marta 2005-2009. Ministerio del Medio Ambiente, Santa Marta-Colombia. 